\title{
Africanisme français et littératures africaines
}

Continuités et discontinuités

French Africanism and African Literature. Continuity and Discontinuity

\section{Kusum Aggarwal}

\section{OpenEdition}

\section{Journals}

Édition électronique

URL : https://journals.openedition.org/etudesafricaines/16554

DOI : $10.4000 /$ etudesafricaines. 16554

ISSN : 1777-5353

Éditeur

Éditions de l'EHESS

\section{Édition imprimée}

Date de publication : 20 novembre 2010

Pagination : 1191-1213

ISBN : 978-2-7132-2252-8

ISSN : 0008-0055

\section{Référence électronique}

Kusum Aggarwal, "Africanisme français et littératures africaines », Cahiers d'études africaines [En ligne], 198-199-200 | 2010, mis en ligne le 02 janvier 2013, consulté le 22 avril 2022. URL : http:// journals.openedition.org/etudesafricaines/16554; DOI : https://doi.org/10.4000/etudesafricaines. 16554

Ce document a été généré automatiquement le 22 avril 2022

(c) Cahiers d'Études africaines 


\title{
Africanisme français et littératures africaines
}

\author{
Continuités et discontinuités \\ French Africanism and African Literature. Continuity and Discontinuity
}

\section{Kusum Aggarwal}

1 L'impérialisme moderne provoque en France dès la seconde moitié du XIX siècle le désir à la fois de conquérir et de dominer des contrées lointaines, et de les saisir sur un mode scientifique dans le but d'estomper les lieux communs embrouillant l'appréhension des sociétés et des cultures non européennes. L'africanisme, en tant que savoir, se situe donc au creux de ces pulsions contradictoires, écartelé dès ses origines entre une volonté de grandeur et de puissance qui galvanise la France empressée de bâtir un empire, et la fascination qu'éprouvent une poignée d'administrateurs coloniaux pour la façon de vivre et de penser des peuples colonisés, les incitant à s'appliquer à les documenter, à les restituer selon les principes de la recherche scientifique.

2 Dans sa critique de l'africanisme, Gérard Leclerc (1972) postule que celui-ci fut effectivement « la fille de l'impérialisme colonial » dans la mesure où il s'est constitué en tant qu'ensemble théorique dès lors que s'impose en Occident le besoin de connaître et de dominer les peuples colonisés. Or, paradoxalement, il peut se concevoir tout autant comme «le père fondateur de la littérature africaine moderne »: il en régit l'avènement non pas seulement à un niveau thématique ou institutionnel, mais, aussi, à un niveau structurel, lui fournissant ce que Bourdieu $(1980: 88)^{1}$ appelle un habitus.

3 Cet essai se donne alors pour objet de cerner les filiations reliant l'africanisme occidental et la production littéraire africaine qui s'enchaînent et s'entrecoupent mutuellement dans un continuum que l'on tend souvent à dénier pour des raisons plutôt idéologiques, souvenances d'une époque révolue qui ne cesse cependant de peser sur l'organisation du champ littéraire africain. L'analyse s'organise ici en trois temps, et s'amorce avec une relecture de l'africanisme occidental de façon à expliciter les raisons qui le conduisent à s'intéresser au fait littéraire africain. La deuxième partie propose 
d'éclairer les modalités dominantes de reprise et de raccommodage par lesquelles les écrivains africains s'approprient les catégories de l'africanisme occidental à leurs usages individuels, et cela justement dans un contexte historique fortement marqué par des revendications anticoloniales comme par une volonté généralisée de contribuer à la construction nationale. Enfin, alors que les années 1990 annoncent le «temps des malheurs » et de «l'ensauvagement» (Mbembe $2000: 269)$ du pouvoir et de l'existence sur le continent noir, l'africanisme ne parvient plus à polariser les écrivains et les penseurs africains, impatients de nouveaux commencements, de l'invention de nouvelles écritures ouvertes sur d'autres mondes.

\section{Jonctions et conjonctions}

4 Élaborant une archéologie des savoirs occidentaux, Michel Foucault rappelait que les limites de l'anthropologie résidaient dans son incapacité de se fonder sur « un concept général de l'homme ", ce qui entravait pour elle, l'accès à " ce qu'il pourrait y avoir de spécifique et d'irréductible en lui, d'uniformément valable partout où il est donné à l'expérience » (Foucault 1966 : 390). Incapable aussi de s'émanciper de ses inconscients culturels pour formuler une idée de l'universel affranchi de la ratio occidentale, cette discipline scientifique à vocation universelle ne saura concilier ses ambitions universalistes avec la relativité des pratiques sociales observées. L'africanisme, configuration disciplinaire conçue dans le dessein de repérer et d'ordonner les traits sociaux et culturels typiques caractérisant les civilisations africaines dans leur singularité, est indubitablement le corollaire de l'éclatement des principes universalistes à l'issue d'une prise de conscience de la pluralité des sociétés humaines. Cependant, une comparaison de la genèse de l'africanisme en Grande-Bretagne et en France durant l'Entre-deux-guerres démontre que l'africanisme comme désignation d'un savoir distinctif, est une construction spécifiquement française. Outre-Manche, un africaniste est un "expert ou spécialiste des affaires, de la culture africaniste " (de L'Estoile 1997 : 20). Deux conceptions de l'africanisme renvoient à deux manières de théoriser les peuples et les cultures africains : à l'instar des travaux de Malinowski, les études sur l'Afrique s'inscrivent au sein de l'anthropologie sociale en Grande-Bretagne pour briguer ainsi une autonomie disciplinaire plus importante alors qu'en France, l'africanisme, évoluant à l'ombre des institutions coloniales dans le seul dessein de contribuer à l'ennoblissement de l'impérialisme français, se caractérise par un dénuement méthodologique et institutionnel.

Pour François Manchuelle (1995), les orientations scientifiques ainsi que les outils et les perspectives de recherche de l'africanisme français découlent largement de la politique d'association prônée par une fraction d'administrateurs coloniaux de gauche conscients de la nécessité d'une colonisation respectueuse des institutions indigènes, les chefs traditionnels pouvant servir d'habiles collaborateurs à leurs homologues européens. Or, Jean-Loup Amselle, quant à lui, met l'accent sur le mode de fonctionnement de l'africanisme français : se situant en droit fil du « multiculturalisme républicain ", ce savoir occidental se déploie, selon lui, en procédant par identification et par isolement des particularismes ethniques, politiques ou culturels à l'intérieur des sociétés colonisées avec l'intention de les fusionner et de les absorber progressivement au sein d'une entité plus homogène au fondement de la construction nationale. Or, appliqué dans les colonies, le principe républicain d'assimilation fonctionne au contraire comme une machine à enfanter des identités, et à les endurcir et les 
excentrer davantage, en les ouvrant à toutes sortes d'exploitation à des fins politiques (Amselle 1996: 106) ${ }^{2}$. Cette opération destinée à confectionner des catégories identitaires et culturelles s'amorce, à bien des égards, avec les travaux du baron Roger, commandant et administrateur du Sénégal et dépendances de 1821 à 1827. Partisan invétéré de l'abolitionnisme et de la colonisation-développement, il réunit une documentation sur les langues et les littératures autochtones ${ }^{3}$ avec l'intention tout à fait estimable de témoigner de l'universalité des civilisations africaines. Cependant, ce n'est que durant la seconde moitié du $\mathrm{xIX}^{\mathrm{e}}$ siècle, sous la gouvernance de Léon Faidherbe, que l'africanisme s'édifie en science empirique dédiée à l'observation des phénomènes socioculturels autochtones afin de se les approprier au service d'une politique coloniale entremêlant "anthropologie raciologique et assimilation républicaine " (ibid. : 117). L'élaboration d'un savoir sur les sociétés africaines pouvait contribuer, à son sens, à mieux manipuler les identités dans une stratégie visant à pourvoir l'administration coloniale de cadres indigènes dévoués à la mission civilisatrice.

6 Ces tentatives d'organiser une documentation sur les sociétés et les cultures africaines se clarifient et s'épurent surtout avec les perspectives et les orientations méthodologiques prônées par Maurice Delafosse, administrateur colonial que l'historien Christophe Wondji considère comme l'un des «fondateurs de la science moderne de l'Afrique $»^{4}$, et dont les travaux reflètent amplement sa formation d'arabisant et d'ethnographe, et sa grande érudition d'historien et de linguiste. Auteur de Haut-Sénégal-Niger (1912), une somme encyclopédique réalisée sur commande officielle à partir d'une documentation substantielle réunie dans le cadre des enquêtes effectuées par des administrateurs coloniaux sur les civilisations africaines, il acquiert rapidement une notoriété remarquable parmi les savants métropolitains : il est sollicité avec Marcel Mauss, Lucien Lévy-Bruhl et Paul Rivet, à collaborer à la fondation de l'Institut d'ethnologie de l'Université de Paris (1925)5. Il réussit aussi à pénétrer les réseaux de recherche britanniques grâce aux bons offices du gouverneur Lugard : celuici le convie à s'associer au projet de création de l'Institut international des langues et des civilisations africaines (IIALC) censé faciliter la mobilité des recherches africanistes entre les divers pôles européens ${ }^{6}$.

7 C'est donc justement dans le cadre de telles recherches effectuées dans le but de saisir le fonctionnement des sociétés et des cultures africaines que se manifeste un intérêt pour les littératures africaines. Le savant français en rassemble les échantillons moins pour elles-mêmes que pour leur utilité documentaire, pour l'éclairage qu'elles sont susceptibles d'apporter sur un fait social ou historique, ou encore sur la conduite des collectivités auxquelles elles appartiennent: car, à sons sens, il est indispensable d'étudier « la mentalité d'un peuple au travers du voile transparent de sa littérature " (Delafosse 2005 : 72). On constate ainsi que la littérature africaine se constitue en un objet de recherche dès lors qu'il est question de substituer au regard extérieur du savant occidental la perspective interne des indigènes considérés désormais comme des médiateurs indispensables pour la légitimation de la recherche occidentale. Toutefois, la reconnaissance de l'autorité du créateur africain ne détraque guère l'organisation du savoir occidental. Nul revirement, nul retournement ne l'atteint. Cela justement parce que, séquestré dans son particularisme d'Africain colonisé, il ne saura jamais être à même de rivaliser avec son homologue occidental. Par ailleurs la dignité littéraire et artistique conférée aux œuvres africaines s'accompagne en définitive de leur exclusion 
des pratiques littéraires dominantes, de leur enfermement dans des régimes critiques parallèles destinés justement à les fixer dans leur spécificité culturelle. S'élaborent donc des règles de lecture et d'appréciation qui reposent pour l'essentiel sur des catégories anthropologiques d'appartenance identitaire et d'authenticité. Ce qui prime est le degré d'africanité d'une œuvre africaine : cette posture critique sera longtemps reprise et retravaillée.

8 Toutefois, à la différence de ses devanciers, de l'abbé Grégoire, auteur d'une anthologie de littérature africaine et du baron Roger, compilateur des fables orales de source africaine, Delafosse n'effectua pas seul une œuvre de praticien satisfait de rassembler des témoignages des cultures africaines. Il se consacra en même temps à un important travail de conceptualisation des littératures africaines dont il élabora une définition systématique et cohérente, interrogeant à la fois ses pratiques, ses institutions, ses modes de différenciation : «Il ne serait pas rigoureusement exact de dire que les nègres ne possèdent qu'une littérature orale et que cette littérature soit nécessairement du genre dit populaire. Sans doute, la littérature orale populaire domine dans l'Afrique noire, mais on y observe aussi une littérature orale savante et une littérature écrite " (Delafosse 2005: 65). On constate à ce propos que, contrairement à l'ethnologue allemand Frobenius plus porté à privilégier une démarche spéculative, Delafosse préconise une approche fondée sur un véritable travail de terrain, sur l'observation empirique et sur la récolte d'informations concrètes, sur le fait littéraire africain notamment. L'influence de Durkheim et de Mauss l'ont incontestablement conduit à être plus vigilant quant à l'importance du document social dans l'élaboration d'un savoir sur le mode de fonctionnement d'une société. Ce choix méthodologique lui servira de caution contre les écueils de certaines thèses culturalistes, notamment celle selon laquelle l'oralité serait une caractéristique exclusive des civilisations africaines.

9 Par ailleurs, en concédant à l'indigène l'autorité ultime du savoir africain, Delafosse entérine son insertion dans le champ scientifique faisant de sa participation un critère de base de la recherche africaniste, déclarant à cet effet que : "Si l'on veut retirer de ces récits toute leur "substantifique moelle", il faut, non pas les lire dans une interprétation faussée par la mentalité du traducteur européen, mais les entendre conter par les nègres eux-mêmes, dans l'une de leurs langues si expressives. [...]. La différence est considérable entre l'effet que produira à cet Européen la lecture d'une traduction, même bien faite, et l'audition de l'original, même dit en une langue qu'il ne comprend qu'imparfaitement» (ibid.: 72). L'Africain colonisé, socialement et politiquement démuni, est désormais propulsé au cœur de la démarche africaniste. Chargé d'attributs culturels, il se voit confié le rôle d'intermédiaire pour être ainsi assimilé et absorbé au profit d'une opération occidentale. Par là ainsi, se débloquent les conditions devant progressivement conduire à l'africanisation de la recherche africaniste. Mais n'imaginons pas que ces mutations parviendront à instituer des rapports égalitaires entre les savants occidentaux et leurs collaborateurs indigènes. Ces derniers sont au contraire relégués au statut de subalterne au service d'une mission scientifique subordonnée à un besoin occidental d'accumulation du capital scientifique (Jezequel 1998). Ce lien organique unissant l'informateur africain au savoir dont il est supposé être un détenteur authentique est un principe fondateur de la démarche africaniste. Il n'est donc pas tout à fait inattendu que ce même principe soit réinvesti et redéployé dès lors qu'il est question de formaliser les conditions de la littérature africaine moderne. Car, comme l'observe Pascale Casanova (1999: 262), «À l'intérieur des espaces démunis, les écrivains sont "condamnés" à une thématique nationale ou 
populaire: ils doivent développer, défendre, illustrer, fût-ce en les critiquant, les aventures, l'histoire et les controverses nationales. Attachés le plus souvent à défendre une idée de leur pays, ils sont donc engagés dans l'élaboration d'une littérature nationale ». L'autonomisation d'une littérature émergente passe donc nécessairement par la conquête d'une indépendance culturelle.

\section{Usages africains de l'africanisme français}

La littérature africaine d'expression française, dans la mesure où elle se situe à l'intersection d'influences multiples, s'ouvre à des imbrications et des enchaînements complexes la conduisant à puiser sans distinction dans les formulations proposées par les discours ambiants, occidentaux et africains. Toutefois, comme la notion de culture africaine occupe une place privilégiée dans le positionnement de cette jeune littérature désireuse de se démarquer et de se différencier des littératures de grand prestige de la France métropolitaine, les écrivains et les penseurs africains étaient nécessairement amenés à questionner les paradigmes du savoir africaniste et les représentations qu'il proposait de la culture africaine, tantôt dans le dessein de les contester, tantôt avec l'intention de les reprendre et de les raccommoder conformément à leur travail d'écrivain.

11 Schématiquement parlant, on peut à cet effet repérer trois grandes tendances correspondant globalement à trois niveaux d'incorporation et d'annexion des modalités du savoir africaniste au profit de la construction d'une littérature africaine. Car si certains empruntent à l'africanisme français leurs théories concernant l'existence d'un substratum africain au fondement de particularismes caractérisant ses formations culturelles et sociales, d'autres y découvrent une praxis susceptible d'éclairer les arcanes des savoirs indigènes réservés seulement à une élite savante ; et d'autres encore le considèrent comme le marqueur de la dépossession et de l'aliénation des intellectuels africains privés du droit à l'autonomie discursive.

À plusieurs égards, la publication de l'Anthologie de la nouvelle poésie nègre et malgache de langue française (Senghor 1948), un véritable monument en l'honneur du panafricanisme, dont Sartre avait bien pressenti la portée révolutionnaire, représente indéniablement un tournant capital dans la prise de conscience de la nécessité impérieuse d'amorcer une réflexion sur les implications de l'émergence d'une littérature africaine et les orientations qu'elle devrait privilégier de façon à contribuer à la reconnaissance des peuples africains et de leurs cultures. Paradoxalement, cet exercice critique, c'est le philosophe français qui le parraine, à sa manière, et faisant appel à la dialectique hégélienne, il échafaude la négritude en la considérant comme l'expression d'une révolte raciale et anticoloniale portée par l'ambition de dénoncer les mécanismes de la violence séculaire que l'Occident n'a cessé d'infliger aux Noirs. En ce contexte de revendication et de contestation, le sujet africain amené à s'identifier pleinement avec sa culture d'origine se donne comme devoir d'œuvrer en vue de la circonscrire, et d'en retracer les pourtours. C'est ce qui explique, selon Sartre, la facture distinctive de la production littéraire africaine : «Feuilletez une anthologie de la poésie blanche d'aujourd'hui : vous trouverez cent sujets divers, selon l'humeur et le souci du poète, selon sa condition et son pays. Dans celle que je vous présente, il n'y a qu'un sujet que tous s'essayent de traiter, avec plus ou moins de bonheur. De Haïti à Cayenne, une seule idée : manifester l'âme noire » (Sartre 1948). 
13 À l'instar de l'existentialiste français, Léopold Sédar Senghor s'attaqua à la tâche d'approfondir et de parfaire l'idée de l'Afrique en lui apposant un contenu repérable, une somme d'éléments constitutifs dénichés ici et là au fil de ses lectures étendues, de ses imprégnations de réseaux diversifiés de penseurs : Karl Marx, Teilhard de Chardin, Henri Bergson, Emmanuel Mounier se côtoient chez lui avec des écrivains et des poètes africains-américains, W. E. B. Du Bois, Langston Hughes, Alain Locke ainsi qu'avec des spécialistes africanistes, Maurice Delafosse, Marcel Griaule, Placide Tempels, Geneviève Calame-Griaule pour en citer quelques-uns. Leurs écrits lui servirent indubitablement à entériner ses intuitions personnelles sur les composantes de la culture africaine et les représentations qu'il conviendrait d'en proposer. On sait, à ce propos, que Senghor avant de théoriser la négritude avait fait école auprès des africanistes français : ayant décroché une agrégation de grammaire en 1935, il suivit des cours de linguistique africaine offerts par Lilias Homburger à l'École pratique des hautes études et ceux d'ethnologie de Marcel Cohen, Marcel Mauss et Paul Rivet à l'Institut d'ethnologie de Paris. De surcroît, il succéda à Delafosse qu'il considérait comme étant «le plus grand des africanisants de France » à la chaire des Langues et civilisations africaines, à l'École nationale de la France d'Outre-mer (L. Delafosse 1976 : 401-402). Il ne tarit pas d'éloges sur ses contributions à la connaissance de l'Afrique : ses conclusions quant aux valeurs définissant la civilisation négro-africaine, ses observations avisées sur le caractère principalement «unitaire» des sociétés africaines, ses découvertes sur le passé prestigieux des peuples africains; tout résonne avantageusement avec sa perception personnelle "des lois de la civilisation africaine", son mode de fonctionnement, totalement irréductible à la civilisation occidentale. La démarche africaniste conduit donc le penseur sénégalais à privilégier la différence : la reconnaissance de la relativité des cultures est désormais un passage obligé pour accéder au droit à l'égalité humaine.

On observe néanmoins que la démarche africaniste que préconise Senghor le rapproche davantage de l'école allemande d'ethnologie africaine, et plus précisément de Léo Frobenius, auteur de l'Histoire des Civilisations africaines que de l'africanisme français plus préoccupé des paramètres méthodologiques des recherches réalisées en son sein. Or, indifférent à la valeur scientifique des observations empiriques et des données documentaires, le poète sénégalais exprime une affinité prononcée avec les thèses essentialistes de Frobenius, et avec, également, ses envolées lyriques sur les correspondances entre les deux civilisations, de l'Allemagne et de l'Afrique noire, qui faisaient pendant à son goût de la poésie romantique allemande imprégnée des charmes du Sturm und Drang. Ainsi, déclarait-il, «Frobenius fut véritablement le moteur spirituel de l'émancipation de l'Afrique noire: sa vision idéaliste d'une Afrique encore pure, non contaminée par les influences extérieures étaient pour nous [...] un aliment qui attisait notre ferveur » (Senghor 1996).

15 L'assignation en définitive d'un contenu précis aux civilisations négro-africaines, la distribution des traits les caractérisant, les cimentant au-delà des diversités évidentes, s'avère indubitablement une entreprise laborieuse amenant l'écrivain à s'immiscer à l'interstice des démarches divergentes dans une tentative parfois de gommer les incongruités, de concilier des tendances antinomiques en vue de faire apparaître l'idée d'une cohérence latente. C'est là, sans doute, une des raisons de sa controverse avec le linguiste Maurice Houis sur la prééminence de l'oralité dans les civilisations africaines. Pour le théoricien de la négritude, il était inadmissible de considérer l'oralité comme un simple fait linguistique: au contraire, elle représentait à ses yeux une 
caractéristique essentielle des civilisations africaines relevant de leur nature fondamentalement distinctive : «L'oralité n'est pas que des langues, mais de toutes les manifestations culturelles négro-africaines. [...] Écriture est synonyme d'abstraction, partant appauvrissement »(Senghor 1959 ; Houis 1971) ${ }^{7}$. Senghor avait donc bien choisi son camp : peu lui importait la dénégation des faits sociologiques et historiques tant qu'il pouvait faire valoir sa conception d'une subjectivité intrinsèquement africaine.

$\mathrm{Au}$ total, l'essayiste sénégalais dérobe à l'africanisme français ses observations sur les sociétés et les cultures africaines, lui empruntant ses concepts de base, et surtout la croyance de l'existence d'un substrat africain soudant ses manifestations culturelles au dépassement des barrières géographiques ou historiques pour légitimer ainsi une vision panafricaniste des civilisations africaines, unifiées en raison de leur homogénéité naturelle. Certes, ses contemplations sur l'esthétique africaine, ses considérations sur le statut et la fonction de l'art dans les sociétés africaines ont largement contribué à façonner l'institution littéraire africaine, à en affirmer l'autonomie. Il faut reconnaître cependant que ses stratégies et ses orientations, Senghor les confectionne moins par un « devoir de vérité » que dans le but de contrecarrer l'hégémonie de l'Occident sur le marché des valeurs symboliques pour y occuper « des positions à partir desquelles les œuvres lues acquièrent une valeur " (Fonkoua 1996).

17 À la différence de Senghor qui extirpe de l'africanisme la matrice des cultures africaines, ce qui captive l'imaginaire de Hampâté Bâ, comme à celui de toute une lignée d'ethnographes autochtones dans la période d'entre les deux guerres mondiales, est plutôt sa dimension pratique, comme démarche méthodologique susceptible de concourir à ressusciter des cultures authentiquement africaines en voie de disparition à la suite de l'intrusion de l'Europe. À l'Afrique composite des poètes de la négritude se substitue ici une Afrique parcellaire, culturellement morcelée, constituée d'unités culturelles disparates, souvent isolées les unes des autres. Une anthropologie indigène adapte et exploite donc les méthodes en vigueur dans l'africanisme français pour pénétrer grâce aux représentants qualifiés des cultures autochtones - les griots, les marabouts et les traditionalistes savants - les sources reculées des savoirs ancestraux qu'elle s'emploie à son tour à rendre à la visibilité en les mettant à l'écrit en traduction française. Hampâté Bâ, quant à lui, a accédé à la démarche africaniste dans le cadre de ses travaux d'auxiliaire de recherche à la section d'ethnologie de l'Institut français de l'Afrique noire. Sa carrière d'écrivain des traditions orales africaines s'amorça lorsque le directeur de l'Institut, le naturaliste renommé Théodore Monod se décida de présenter, à l'occasion de la séance inaugurale du Congrès international des Africanistes en 1950, sa version du conte initiatique peul Kaïdara, ostensiblement recueilli auprès de traditionalistes diligents. Une négritude pondérée, enfantée en colonie sous la surveillance des instances coloniales, ne pouvait guère aspirer à une meilleure consécration.

18 Ses premiers écrits - L'Empire peul du Macina (1955), Koumen (1961), Kaïdara (1969), L'Éclat de la grande étoile (1974) - s'inscrivent pleinement dans la mouvance africaniste esquissée par Marcel Griaule, auteur de Dieu d'eau, fruit de conversations avec son informateur dogon, Ogotemmêli. Cependant, Hampâté Bâ est loin d'être inconscient des limites de l'africanisme français: un savoir mécaniste reflétant à sa manière les inégalités de la société coloniale : « Le commandant de cercle donna l'ordre à Alfa Maki Tall, chef de canton de Bandiagara, d'envoyer à Equilbecq tous ceux qui connaissaient des contes : hommes, femmes, vieillards ou enfants. Chaque conte retenu était payé 10, 
15 ou 20 centimes, selon sa valeur et sa longueur. Wangrin fut détaché pour être l'interprète d'Equilbecq, mais très rapidement ce dernier le chargea de recueillir directement les contes pour lui. Wangrin écoutait, prenait des notes, rédigeait la traduction en français puis les remettait à Equilbecq, qui y apportait la dernière main » (Heckmann 1987 : 219).

Ces tensions au sein de l'africanisme colonial conduisent l'écrivain africain à se repositionner de façon à opérer ce retournement nécessaire pour transiter du statut d'informateur subalterne au statut d'auteur autonome. À cette fin, il effectue des manipulations éditoriales en vue d'écarter ses anciens collaborateurs, de gommer les traces de leur partenariat. Significativement, la deuxième édition de Tierno Bokar, le Sage de Bandiagara (1980), n'a plus comme co-signataire Marcel Cardaire, officier chargé des Affaires musulmanes, qui en avait facilité la publication; il en est de même avec les éditions successives de Kaïdara dont il s'octroie la qualité d'auteur. Il se met d'autre part à se distancier progressivement de l'épistémè à l'œuvre dans l'africanisme français dont la construction repose, malgré toutes ses tentatives de percer la conscience indigène, sur une relation d'extériorité avec celle-ci. Au fait, s'il s'emploie, dès l'origine, à étayer et à accommoder les composantes d'une culture peule, c'est moins pour répondre aux nécessités d'un savoir occidental que pour son désir personnel de se saisir de l'expérience collective des peuples africains. Ressusciter et remémorer les éléments de ces traditions ancestrales a pour fonction de jeter les bases d'une bibliothèque proprement africaine. Par ailleurs, l'africanisme français dans la mesure où il privilégie la documentation des faits collectifs - les propos d'Ogotemmêli ne sont dignes d'intérêt que parce qu'ils reflètent les comportements sociaux spontanés d'un groupe ethnique $^{8}$ - n'est guère en mesure de favoriser véritablement l'éclosion d'une littérature africaine dont l'existence implique avant tout que l'on reconnaisse la possibilité d'une véritable subjectivité africaine, irréductible aux ordonnances de la communauté. Son roman, L'Étrange destin de Wangrin (1973), tout comme ses mémoires, Amkoullel l'enfant peul (1991) et Oui mon commandant! (1994), manifestent évidemment un cogito autre que celui qui avait motivé la construction de l'africanisme français : il est le fait de la trajectoire individuelle d'un sujet autonome.

En contrepartie des tenants de l'africanité qui, le déformant et le détournant, épuisent l'africanisme occidental dans une stratégie correspondant à des fins personnelles, l'œuvre de V.Y. Mudimbe dérive de l'exigence de questionner, dans une perspective critique, les principes au fondement du discours occidental, anthropologique ou littéraire, dans une tentative de circonscrire ses répercussions sur les conditions d'un savoir proprement africain. Le philosophe zaïrois passe alors au crible une somme impressionnante de textes de missionnaires, de voyageurs et de penseurs occidentaux, d'Hérodote à Foucault, Sartre et Lévi-Strauss sans oublier Griaule, Tempels et Delafosse avec l'objectif de saisir leur démarche devant la question de l'altérité : si oui ou non ils seraient à même de fournir au chercheur africain un lieu d'inscription pour ses recherches scientifiques. Car à ses yeux, toute l'ambiguïté du savoir occidental découle du fait qu'il ne peut entretenir avec l'altérité une relation purement théorique : celui-ci est en même temps affublé de traits caractéristiques qui renvoient davantage aux modalités de son existence. La question est donc de savoir quelle attitude adopter face au savoir occidental puisqu'il fixe le chercheur africain dans son africanité, lui déniant toute liberté d'être et de penser: "Je ne suis plus qu'un symbole noir enveloppé de draps blancs. Un totem » (Mudimbe 1979: 107), crie de frustration Nara, le jeune 
historien noir, incapable de transcender les cloisonnements d'un savoir occidental pour accéder au statut de sujet autonome.

21 Ce questionnement de l'épistémè à l'œuvre dans la pratique scientifique occidentale n'est aucunement une opération gratuite. Il est, au contraire, le fruit de la réflexion amorcée au cours des années 1970, dans un contexte où il devenait urgent de délibérer sur l'avenir des universités africaines, comme sur les conditions et les finalités de l'activité scientifique en Afrique, d'autant que se détachaient, à la même époque, de nouvelles tendances scientifiques impulsées par l'influence croissante des thèses africanistes et afrocentristes formulées dans le cadre de la négritude et des travaux de Cheikh Anta Diop et de ses disciples. Ces travaux revendiquent la relativité de la pratique scientifique africaine, et se donnent pour première fonction la démobilisation des lieux de pouvoir occidentaux. Or, la science africaine peut-elle avoir comme principal objet de nier ou d'affirmer les catégories de la pensée occidentale? On constate, à cet effet, que la lecture et la relecture des textes édifiant un ordre du savoir africain auxquelles se consacre inlassablement le philosophe zaïrois sont motivées par la nécessité de prendre acte des leurres d'une science au service des idéologies, qu'elles soient africaines ou occidentales, car "Cette misère intellectuelle [...] donne à l'Africain producteur de "science" l'air d'un être non caractérisé par l'autonomie, celle-ci étant entendue au sens étymologique, c'est-à-dire "comme la propriété des comportements qui sont eux-mêmes leurs propres normes" [...]. Le chercheur africain est ou fixé sur un culte de la différence qui le bloque complètement et l'empêche de dire, en vérité, ses raisons d'être, son insertion dans les rapports complexes de domination; ou enfermé volontairement dans un vicieux cercle où il assume un rôle à la fois d'objet et de sujet : réifié par l'expérience du regard d'autrui et les techniques de sa parole, il actualise un comportement hétéronome visant ses œuvres et son travail comme des contraintes d'un déterminisme aveugle exigé par "la Science" » (Mudimbe 1973 : 119).

En somme, le rude combat que mène inlassablement Mudimbe a pour objet d'entrevoir les conditions nécessaires pour dessaisir la recherche africaine de l'intrusion à la fois de l'idéologie coloniale et de l'idéologie nationale qui tendent à favoriser une perspective indigéniste, la réduisant en un exercice destiné à promouvoir les valeurs identitaires de race et de nation. Or, la construction d'un savoir véritable se réalise, estime-t-il, en dehors des lieux consacrés de l'histoire, en dehors aussi bien des pressions du monde social : elle est le fait d'un regard pur, d'une sorte de "degré zéro », point de départ de l'exercice de la science qui est donc «ouverture critique, dynamique, pratique scientifique mobile, féconde, totalisante, se réfléchissant dans la vie et dans les contradictions réelles d'une formation sociale "(ibid.). Au terme de son périple à travers les limbes de la pensée occidentale, le chercheur africain s'ouvre à une conscience de l'autonomie du sujet africain qui existe en deçà du discours réifiant de l'Occident, et est donc libre d'opérer ce retournement nécessaire pour réactiver à sa façon un savoir sur l'Afrique, car «It has also become obvious, even for this subject, that the space interrogated by the series of explorations in African systems of thought is not a void» (Mudimbe 1988 : 200).

23 Ayant reconquis l'autonomie scientifique, il retourne sur un domaine de savoir pour lequel il éprouvait un intérêt bien avant sa crise intellectuelle à Paris au lendemain de mai 1968 et entreprend une exégèse des paraboles et des fables des Luba pour achever ainsi le décentrement d'une approche accumulée au contact du savoir occidental (ibid. 1991). Sur ce plan, l'itinéraire intellectuel de Mudimbe présente incontestablement des 
recoupements avec celui de son homologue béninois, Paulin Hountondji, disciple de Husserl, reconnu pour sa vibrante critique de l'ethnophilosophie dans un essai devenu un classique : Sur la "philosophie africaine » (1976); il bifurque dans Les Savoirs endogènes. Pistes pour une recherche (1994) vers une recherche portant sur les systèmes du savoir africains.

Ces trois itinéraires illustrent ainsi les possibilités de l'africanisme français, faisant voir, comme le dirait Bourdieu (1980: 94) les «potentialités objectives» inscrites dans «les structures cognitives et motivatrices qui sont constitutives de l'habitus ». Par là ainsi, on constate que, en dépit de ses limites, idéologiques ou autres, l'africanisme réunit les conditions adéquates pour un revirement épistémologique afin de libérer ainsi un processus de réhabilitation et de revitalisation culturelle et scientifique dans les colonies africaines soucieuses d'accéder à la dignité littéraire. Ces trois écrivains s'assignent effectivement la mission d'investir des postures d'africanité et s'inscrivent pleinement dans un espace africain avec le but de la faire parler. Ils entendent donc édifier ainsi les conditions d'une "révolution herderienne " propice à une renaissance littéraire et artistique9.

\section{L'africanisme décroché}

Les années 1990 instaurent un processus de décrochage et de mise à distance à l'égard de l'africanisme : celui-ci n'inspire plus un engouement identique parmi les écrivains et les penseurs africains impatients de nouvelles postures conceptuelles et méthodologiques plus à même de décoder le sens des mutations qui implosent les sociétés africaines. Celles-ci ne s'apparentent plus aux sociétés ahistoriques, enclines à l'immobilisme, ainsi que l'anthropologie l'avait subodoré ; au contraire l'histoire parait y avoir pris une ampleur ahurissante les laissant exsangues, totalement exténuées. L'Afrique noire, dont on se demandait si elle était mal partie, n'est-elle pas, désormais, faite pour trépasser, abandonnée au cercle infernal de la paupérisation, du sida, des famines, devant l'indifférence de l'État devenu symbole de dévoration et de violation? Cette Afrique en gestation, prégnante de phénomènes inusités, faut-il l'entrevoir au prisme de la déviance et de l'anormalité, de l'« étrangeté et du monstrueux » (Membe 2000 : 19) comme on serait tenté de le faire ? Ou est-elle encore une réalité traduisible, accessible à la raison humaine? Si oui, dans quel langage? Ce questionnement qui sommeille au creux des romans africains depuis Le devoir de violence (1968) de Yambo Ouologuem, et Les Soleils des Indépendances (1970) d'Amadou Kourouma, atteignit son point d'orgue avec les travaux et les recherches de l'historien et du philosophe camerounais Achille Mbembe qui, à la suite d'importantes recherches sur Ruben Um Nyobè, fondateur de l'Union des populations du Cameroun (UPC) et chef de fil du mouvement indépendantiste dans le Sud-Cameroun, publia un essai novateur sur l'imagination politique dans l'Afrique contemporaine, une tentative de rendre compte des conditions nécessaires pour saisir l'Afrique comme objet de recherche dans ce contexte précis de son éboulement vertigineux vers l'anéantissement.

Cependant, la démarche scientifique du savant camerounais n'est pas sans rapport avec son itinéraire personnel empreint de l'expérience du désenchantement, de la dislocation, ou encore du bannissement tout court escamotant toute perspective de retour au pays natal. Ce dernier ne provoque guère plus de nostalgie comme chez les générations précédentes, mais un profond désarroi, une conscience douloureuse de ce 
que Mbembe appelle le « malheur généalogique » condamnant à jamais les intellectuels africains à l'exode et à l'" ex-patriation ", et surtout à se brouiller avec leur patrie et leur histoire, comme avec eux-mêmes (Mbembe 1993 : 81, 87). Réduit à l'impossibilité de s'identifier avec cette Afrique, complice discrète d'un ordre politique et social vivotant entre l'arbitraire et l'injustice, l'écrivain se trouve contraint à adopter une écriture de l'écart et de la faille; une écriture alors purifiée de rattachements et de loyalismes territoriaux ou identitaires.

Cette mise à distance par rapport au continent noir paraît être pour l'historien africain une condition essentielle pour une meilleure prise en compte de l'objet de recherche confronté à un double écueil : d'une part du binarisme constituant la trame à la fois du discours occidental et du discours africain voués à la fabrication de l'altérité; et, d'autre part, de l'instrumentalité du savoir africaniste, creuset des angoisses et des obsessions du sujet regardant : «Il n'y a presque jamais de discours sur l'Afrique pour elle-même "(ibid.), fulmine Mbembe. Les outils et les méthodes nécessaires qu'il s'octroie pour mettre un terme à ce "long sommeil dogmatique ( (ibid. $2000: 10$ ) qui assomme le savoir africaniste le conduisent donc à opérer un retournement épistémologique, et à arracher la recherche africaniste aussi bien de la problématique de l'identité et de la différence qui l'a entraînée dans une impasse, que des théories du postmodernisme et du postcolonialisme, éprises de représentations discursives et d'abstractions conceptuelles, pour la rattacher davantage aux faits sociaux à proprement parler. Jouissant d'une formation en histoire et en science politique, Mbembe était sans doute mieux habilité que Mudimbe et Hountondji à recentrer l'étude des sociétés africaines sur leur propre historicité, à savoir sur "les fondements de ce que l'on pourrait appeler leur "légalité propre", leurs "propres raisons d'être" et leur "rapport à rien d'autre qu'à elles-mêmes" » (ibid. : 14).

On aura compris dès lors le raisonnement du philosophe africain: le désordre qu'engendrent les sociétés africaines, les violences, les privations et les avilissements qu'elles génèrent ne sauraient se concevoir comme des indices de leur inachèvement, ou de leur irréductibilité à la raison humaine. Il renvoie au contraire aux comportements de ces sociétés qui produisent elles aussi du sens, puisque «la réalité sociale en Afrique subsaharienne est un ensemble de pratiques socialement produites, matériellement codifiées et symboliquement objectivées " (ibid.: 16). Mais, comme ces pratiques sont structurées ici, comme ailleurs, à partir de l'expérience qu'en effectuent des acteurs sociaux, il est indispensable de circonscrire les modalités de fonctionnement du sujet africain dont l'autonomie est pour Mbembe un fait qui va de soi : «La constitution du soi africain comme sujet réflexif passe aussi par le faire, le voir, l'ouïr, le goûter, le sentir, le désir ou le toucher. Aux yeux de tous ceux qui sont impliqués dans la production de ce soi et de ce sujet, ces pratiques constituent ellesmêmes ce que l'on pourrait appeler des expressions humaines significatives » (ibid.). Par là, le philosophe africain restitue au sujet africain sa capacité d'individualité : celuici s'accomplit à travers l'exercice de son existence "qui vit et épouse sa contemporanéité » (ibid. : 38). Cette contemporanéité de la postcolonie est, par ailleurs, constituée d'une pluralité d'espaces publics soumis à une pluralité de temporalités «qui recèlent en elles la possibilité de trajectoires fractionnées, ni convergentes, ni divergentes, mais imbriquées, paradoxales » (ibid.: 37). Le sujet postcolonial mobilise dès lors une pluralité d'identités qui sont elles aussi fluctuantes, négociées incessamment dans le but d'une meilleure efficacité sociale. La postcolonie, lieu d'improvisations perpétuelles, se situe donc au dépassement de la dyade universalisme- 
relativisme, car de l'avis de Mbembe, en postcolonie, le particularisme et l'universalité s'enchaînent et se recoupent : tout particularisme recèle en lui une part d'universalité.

En théorisant la postcolonie, Mbembe signe l'achèvement de l'africanisme dans la mesure où il déloge l'idée d'une Afrique signifiante, génératrice de comportements déviants, rendant inévitable une redistribution de rôles et de tâches au détriment du chercheur autochtone considéré jusque-là comme étant le dépositaire privilégié du savoir sur l'Afrique. Toutefois, ces tentatives d'autonomiser la recherche africaniste, de l'ouvrir à des pratiques et des comportements nouveaux, ne suscitent pas un enthousiasme identique parmi les chercheurs africains. En témoignent, le passage de Mbembe au Codesria, de 1996 à 2000, et la réaction de l'establishment scientifique africain à son égard (Amselle 2008 : 90-94).

On constate aussi de semblables mutations dans la littérature africaine contemporaine où surgissent de nouveaux questionnements, de nouveaux comportements littéraires portés à la désafricaniser, à la déterritorialiser de façon à la connecter avec de nouveaux lieux, de nouvelles pratiques littéraires dans le but sans doute d'élargir le cadre des concurrences littéraires. L'œuvre de l'écrivain congolais, Alain Mabanckou, est à cet égard emblématique de nouvelles stratégies de positionnement. Se branchant sur le double espace intellectuel du monde littéraire français et du monde universitaire américain, lieux majeurs de diffusion et de consécration des littératures francophones, qui lui assure une relative indépendance par rapport aux institutions littéraires françaises, on peut aisément concevoir d'autres inscriptions littéraires. Écrire, c'est donc pour lui déconstruire des identités, les multiplier à l'infini à la croisée d'hybridations ondoyantes, par la mise en relation avec des littératures lointaines de façon à se les approprier en vue d'accoucher d'une nouvelle littérature africaine. Le parcours du héros de Black Bazar, dandy invétéré, maître ingénieux de l'art de l'habillement, divulgue en effet les tensions intrinsèques du champ littéraire africain et les ambitions qui l'animent. S'éveillant par la force des choses à ses inclinations naturelles pour la vocation d'écrivain, il évoque, sans aucune culpabilité, et plutôt avec un humour truculent, les affres de ses origines africaines: «Je ne suis pas peureux, je ne manque pas de courage et de volonté. C'est une question de stratégie: un lâche vivant vaut mieux qu'un héros mort. C'est un conseil très judicieux de mon défunt oncle qui avait déserté le camp militaire durant la guerre du Biafra pour défendre sa modeste personne, mourir plutôt de mort lente que pour des idées qui n'auront plus cours quelques lustres plus tard [...]. Je me suis rendu compte que la désertion est héréditaire dans ma famille puisque moi aussi j'ai fui les obligations dans mon pays d'origine. Moi les armes etcetera, ce n'est pas mon truc » (Mabanckou 2009 : 178-179).

31 Pour le surnommé Fessologue, les liens de sociabilité se desserrent et se relâchent progressivement : sa compagne africaine l'abandonne, les siens le quittent et, esseulé, il s'attache à sa passion d'écrire: mais qu'est-ce écrire? Qu'est-ce être écrivain? Où s'inscrire? Au fil des rencontres et des contacts se précise une idée de ce qu'est le travail d'écrire : c'est « savoir organiser mes idées au lieu d'écrire sous l'impulsion de la colère et de l'aigreur " (ibid. : 169); mais c'est aussi prendre acte de la nécessité des solidarités sociopolitiques, diasporiques et panafricanistes. La rencontre d'immigrés algériens, haïtiens et antillais cristallise en lui la conscience que l'idée d'une particularité africaine est avant tout un mythe, qu'elle représente au fait une pluralité d'intérêts et de positions souvent irréconciliables. Quoi qu'il en soit, il parvient au terme de son parcours à donner un sens à sa vocation d'écrivain lors d'un entretien 
dans un café parisien avec un écrivain breton, désireux, lui, d'imbrications et d'entrecroisements avec des mondes lointains porteurs d'autres vies et d'autres savoirs. Interloqué par cet intérêt porté à son égard, l'écrivain apprenti accède dès lors à l'existence: écrire c'est donc un geste de mise en relation avec le lointain. Il se produit en lui une profonde mutation intérieure qui se matérialise par un reniement catégorique des attachements de race et de classe puisque écrire, c'est s'individualiser, se distinguer : "Je défrise mes cheveux et les tire en arrière comme dans les films des années trente ou quarante [...]. Je dois me distinguer, créer mon propre style même à contre-courant. [...] L'homme est le boulanger de sa vie. Donc c'est à moi de pétrir mon corps, de le transformer comme je l'entends, un point c'est tout" (ibid.: 225). S'arrachant petit à petit des conventions sociales qui le condamnaient à une fixité identitaire, à un immobilisme national et culturel, il se résout dès lors à se défaire de l'africanité.

Pour s'imposer dans le monde de l'écriture, le Fessologue aura à se détacher des mondanités de ses compatriotes: l'art est un lieu de la dénégation du monde social. Tout comme il est question de goût et de discrimination esthétique, « l'écrivain est un artiste, c'est un peintre des mots» (ibid.: 232). C'est un univers distinct régi par ces règles différentes : « Le vrai peintre est celui qui transgresse les normes » (ibid. : 236). Il secrète ses propres valeurs sans grand rapport avec les valeurs sociales. S'arrachant à une logique communautaire qui ramène toute œuvre d'art à sa dimension sociale, il revendique une liberté de l'artiste, celle de créer ses propres solidarités, ses propres filiations représentant une pluralité d'influences. Car sa bibliothèque personnelle rassemble sans distinction René Magritte, Miles Davis, Compay Secundo, Béatrix Beck, Georges Simenon, Maurice Maeterlinck, Amélie Nothomb, Dominique Rolin. Le choix d'une telle palette d'écrivains et d'artistes est révélateur de la stratégie de l'écrivain congolais dont les goûts sont davantage le fait du principe d'intertextualité et d'intersection que des critères d'adhésion nationale ou culturelle : «Entre le Nigérian Wole Soyinka et le Français Louis-Ferdinand Céline, par exemple, un des deux est pour moi un "étranger". Et ce n'est surtout pas Céline que j'ai lu dans le texte, mais Soyinka que j'ai découvert par la voie détournée de la traduction. On pourrait polémiquer sur le fait que Soyinka et moi "possédons" l'Afrique ou sommes "possédés" par l'Afrique, notre continent, notre terre natale. Il s'agirait là d'un autre débat, et une telle conception "continentale" des lettres a longtemps nui à l'intelligibilité de l'art et à son indépendance. L'Afrique, vaste continent, enchevêtrement de cultures, affiche une telle complexité que l'on pourrait dénicher deux écrivains d'un même pays qui seraient "étrangers" l'un à l'autre! Étrangers dans leur vision du monde, étrangers dans leur manière d'entendre le chant de l'oiseau, étrangers dans leur façon d'aller rencontrer l'art » (ibid. $2007: 61$ ).

Ce travail d'amplification et d'extension de l'habitus structurant la littérature africaine désireuse d'accéder à de nouveaux territoires, de conquérir de nouvelles frontières, conduit Léonora Miano à se brancher sur la diaspora africaine pour retourner ainsi sur les lieux d'une mémoire longtemps occultée en évoquant la complicité des peuples africains dans le commerce triangulaire, mais aussi avec l'intention de s'enchaîner sur d'autres littératures pour faire de son œuvre le lieu d'identifications multiples. Son troisième roman, Tels des astres éteints (2008a), revendique une intertextualité intime avec la musique et la littérature africaines-américaines : à la manière des comédies musicales américaines, le récit se déroule au rythme d'une sélection de compositions de jazz et de blues et élabore une réflexion sur les conditions actuelles du racisme en 
France pénétrées d'influences multiples, diasporiques ${ }^{10}$. Cependant, ce n'est pas ici encore un nouveau réquisitoire contre le racisme des Blancs, mais une critique sans compromission des nouvelles orthodoxies qui paralysent les Noirs dans le repliement communautaire. En somme, la question que pose l'écrivaine camerounaise est de savoir : qu'est-ce que le "fait d'être Noir »? Ou plus exactement quelle conscience les Noirs ont-ils de leur situation dans le monde ? Quelle est leur part dans leur expérience de l'exclusion? Le racisme est-ce toujours un fait de l'autre? Ou comme le constate l'historien français Pap Ndiaye (2008: 49) : «Les Noirs ont en commun de vivre dans des sociétés qui les considèrent comme tels. Ils n'ont pas le choix d'être ou de ne pas être tels qu'on les voit. Ils ont en revanche le choix d'assumer leur identité racialisée, ou de la rejeter comme impropre à leur être profond. Ce choix n'est pas susceptible de modifier radicalement la prescription raciale. Pour paraphraser les propos de Sartre concernant les juifs, un Noir est un homme que les autres hommes tiennent pour noir. »

L'immigration, ainsi que l'auteur le décrit, conduit à la ghettoïsation de l'immigré réduit à l'invisibilité, telle la communauté noire décrite dans le roman construit sur l'anéantissement de l'autre, du Blanc réduit lui aussi à l'invisibilité : les Blancs sont pour tout dire un fait de discours et déterminent à distance le comportement des Noirs. Les trois personnages au cœur du récit sont représentatifs chacun à leur façon d'une idéologie africaine : Amandla, métisse des îles, est d'un radicalisme afrocentriste hérité de Marcus Garvey et de Bob Marley et pense que la réunification des " kémites » est indispensable à leur régénération ; Shrapnel, un orphelin des quartiers miséreux entré clandestinement en France, se situe lui plutôt du côté des africanistes dans la mesure où il est imprégné des idées sur ce qu'est un être-dans-le-monde-africain ; et enfin Amok, un nihiliste des classes aisées, est prisonnier des mystifications faciles : la France est pour lui un prétexte de fuite hors de son roman familial, et de son lourd passé colonial dont il n'arrive pas à démêler les fils. Ces immigrés, emmurés sur eux-mêmes, névrotiques, se métamorphosent et se transmuent progressivement au fil des rencontres et des déplacements qui jalonnent leur parcours en confrontation aussi bien avec d'autres consciences noires sur la question raciale. En somme, l'idée de passer au crible une multitude d'identités noires, fluctuantes, éclatées, et qui ne parviennent pas à communiquer, et encore moins à forger un langage cohérent, c'est sans doute les porter à la visibilité, mais c'est aussi les démystifier : l'enfer est-ce vraiment l'autre? C'est en fait, la question que pose le roman par le truchement de ce périple à travers la conscience labyrinthique de ces trois personnages qui ne vivent que par leurs pensées.

S'ils ont tous les trois des identités mobiles, Amok est le seul à réussir à s'arracher de ses origines: "Le pays c'était l'enfance. C'était la source, pas la destination. L'intra muros apportait l'apaisement [...]» (Miano 2008a : 353). Il découvre que : «Pour aimer, il faut d'abord exister. On ne pouvait envisager de rencontrer l'autre sans être rien soimême " (ibid. : 360). Il conclut dès lors que les Africains sont les premiers responsables des frontières qui les excluaient : «Les chaînes qu'on avait soi-même forgées étaient les plus difficiles à ôter. Elles définissaient celui qui les portait. Sans ces entraves intimes, il ne reconnaissait plus le monde. Ce n'était plus le moment d'envisager une thérapie. De toute façon, ce qu'il y avait en lui de profondément subsaharien lui intimait l'interdiction formelle d'aller raconter sa vie à un étranger [...]. Il devait au préalable cesser d'être le fils de ses parents. Devenir un homme » (ibid. : 289). S'inspirant donc de la perspective de l'écrivain africain-américain, James Baldwin qui, en pleine période de la ségrégation raciale aux États-Unis dans les années 1950, eut l'audace de plaider en 
faveur d'une éthique de l'être, l'écrivaine camerounaise convie à une prise en compte des pièges des idéologies de l'africanité et de l'afrocentrisme, en vue de libérer les possibilités de configurations nouvelles. Car, aux yeux de Miano, l'avenir est à ceux qui savent s'inventer, et se constituer ailleurs que là où ils sont attendus comme le laisse entendre l'homme de demain qui sera lui l'Afropéan : «L'identité était un processus, un mouvement constant, pas une stèle à trimballer sur le dos. Il était déjà assez difficile d'être un humain [...]. Les gens étaient des individus pas les particules indifférenciées d'une masse [...]. Des années durant il s'était imaginé être une conscience, plutôt qu'un corps. Il s'était vécu de l'intérieur » (Miano 2008b : 54).

De l'africanisme occidental à l'édification d'une littérature africaine, et de là aux tentatives actuelles de créer une littérature-monde ouverte sur les lointains inconnus de mondes à découvrir, à construire, ces jeunes littératures longtemps défavorisées semblent désormais avoir atteint un stade de pleine maturité au point de renier la dernière forme de dépendance qui les connectait à un territoire et à une culture. Cela dit, on ne saurait pas non plus attribuer le dépérissement de l'africanisme occidental au seul fait de la délégitimation aux mains des chercheurs successifs: les conditions actuelles sur le continent noir ne sont plus guère propices au travail de terrain ainsi que c'était le cas dans les années 1930-1940, âge d'or de la recherche française. En revanche, en s'éloignant de l'Afrique et des catégories de pensée qui lui sont associées, les écrivains et les penseurs paraissent désormais s'éveiller à de nouvelles écritures inspirées de nouvelles postures critiques, de nouveaux retournements historiques : « Ce qui peut être perçu comme situation d'exil peut tout autant être considéré à travers la catégorie de l'“arrivée" »"11 remarquait à ce sujet Bernard Mouralis (2007: 95). La question est cependant de savoir dans quelle mesure cette « arrivée » contribuera-t-elle à réordonner les principes structurant le champ scientifique et littéraire de façon à abolir en définitive les partages qui y régissent.

\section{BIBLIOGRAPHIE}

AGGARWAL, K., 1999 Amadou Hampâté Bâ et l'africanisme. De la recherche anthropologique à l'exercice de la fonction auctoriale, Paris, L'Harmattan.

-, 2007 « Présentation », Kelédor, du Baron Roger, Paris, L’Harmattan (« Autrement mêmes »).

-, 2008 « Présentation », Fables sénégalaises, du Baron Roger, Paris, L'Harmattan (« Autrement mêmes »).

AMSELLE, J.-L., 1996 Vers un multiculturalisme français. L'empire de la coutume, Paris, Aubier.

-, 2008 L'Occident décroché. Enquête sur les postcolonialismes, Paris, Stock.

AMSELlE, J.-L. \& SIBEUD, E., 1998 Maurice Delafosse. Entre orientalisme et ethnographie : l'itinéraire d'un africaniste (1870-1926), Paris, Maisonneuve et Larose.

BÂ, H. A., 1957 Tierno Bokar, le Sage de Bandiagara (avec la cosignature de M. Cardaire), Paris, Présence Africaine. 
-, 1961 Koumen, texte initiatique des pasteurs peul (en coll. avec G. Dieterlen), Paris, Mouton.

-, 1962 [1955] L'Empire peul du Macina (avec la cosignature de J. Daget), Paris, Mouton.

-, 1969 Kaïdara, récit initiatique peul (en coll. avec L. Kesteloot), Paris, Julliard (« Les Classiques africains »).

-, 1973 L'Étrange destin de Wangrin, Paris, UGE, 10/18.

-, 1974 L'Éclat de la grande étoile (en coll. avec A. Ibrahim Sow \& C. Seydou), Paris, A. Colin (« Les Classiques africains »).

-, 1978 Kaydara (avec des illustrations de F. Hanes), Abidjan, Nouvelles éditions africaines.

-, 1980 Vie et enseignement de Tierno Bokar. Le Sage de Bandiagara, Paris, Éditions du Seuil.

-,1991 Amkoullel, l'enfant peul, Arles, Actes Sud/ACCT.

-, 1994 Oui mon commandant!, Arles, Actes Sud/ ACCT.

BAZIN, J., 1985 « À chacun son bambara », in J.-L. AMSELlE \& E. M’BOKоLo (dir.), Au cœur de l'ethnie. Ethnies, tribalismes et État en Afrique, Paris, La Découverte : 87-125.

BOURDIEU, P., 1980 Le sens pratique, Paris, Éditions de Minuit.

CASANOVA, P., 1999 La République mondiale des Lettres, Paris Éditions du Seuil.

DELAFOSSE, L., 1976 Maurice Delafosse. Le Berrichon conquis par l'Afrique, Paris, Société française d'histoire d'Outre-mer.

DELAFOSSE, M., 2005 Les Nègres (présentation de B. Mouralis), Paris, L'Harmattan (« Autrement mêmes »).

FONKOUA, R., 1996 « L'Afrique en Khagne. Contribution à une étude des stratégies du discours dans le champ littéraire francophone », Présence Africaine, 154 : 173-174.

FOUCAUlT, M., 1966 Les mots et les choses. Une archéologie des sciences humaines, Paris, Gallimard.

GILROY, P., 1993 The Black Atlantic. Modernity and Double Consciousness, Cambridge, Harvard University Press.

HECKMANN, H., 1987 « Genèse d'un livre : L'Étrange destin de Wangrin », Nouvelles du Sud, 6-7 : 219-225.

HOUIS, M., 1971 Anthropologie linguisitique de l'Afrique noire, Paris, Presses universitaires de France. HOUNTONDJI, P. J., 1976 Sur la « philosophie africaine », Paris, Maspéro.

-, 1994 Les Savoirs endogènes : pistes pour une recherche, Dakar, Éditions du Codesria.

JEZEQUEL, J.-H., 1998 « Maurice Delafosse et l'émergence d'une littérature africaine à vocation scientifique », in J.-L. AMSELLE \& E. SIBEUD (dir.), op. cit. : 90-104.

KOUROUMA, A., 1970 Les Soleils des Indépendances, Paris, Éditions du Seuil.

LECLERC, G., 1972 Anthropologie et colonialisme. Essai sur l'histoire de l'africanisme, Paris, Fayard.

DE L’ESTOILE, B., 1997 « Africanisme et Africanism. Esquisse de comparaison franco-britannique », in E. SIBEUD \& A. PIRIOU (dir.), Africanisme en questions, Paris, Éditions de l'EHESS, Centre d'études africaines (« Dossiers africains ») : 19-42.

MABANCKOU, A., 2001 « Le chant de l'oiseau migrateur », in M. LE BRIS \& J. ROUAUD (dir.), Pour une littérature du monde en français, Paris, Éditions du Seuil : 55-66. 
-, 2009 Black Bazar, Paris, Éditions du Seuil.

MANCHUELLE, F., 1995 « Assimilés ou patriotes africains ? Naissance du nationalisme culturel en Afrique française (1853-1931), Cahiers d'Études africaines, XXXV (2-3), 138-139 : 333-368.

MBEMBE, A., 1993 « Écrire l'Afrique à partir d'une faille », Politique africaine, 51 : 69-97.

-, 2000 De la postcolonie. Essai sur l'imagination politique dans l'Afrique contemporaine, Paris, Karthala.

MIANO, L., 2008a Tels des astres éteints, Paris, Plon.

-, 2008b Afropéan Soul et autres nouvelles, Paris, Flammarion.

MOURALIS, B., 2007 « Le romancier africain et "L'énigme de l'arrivée" ", in H. CHAMPION (dir.), L'illusion de l'altérité. Études de littérature africaine, Paris, Honoré Champion : 95-108.

MUDIMBE, V. Y., 1973 L'Autre face du royaume. Une introduction à la critique des langages en folie, Lausanne, L'Âge d'Homme.

-, 1979 L'Écart, Paris, Présence africaine.

-, 1988 The Invention of Africa: Gnosis, Philosophy and the Order of Knowledge, Bloomington, Indiana University Press.

-, 1991 Parables and Fables. Exegesis, Textuality and Politics in Central Africa, Madison, University of Wisconsin Press.

NDIAYE, P., 2008 La condition noire. Essai sur une minorité française, Paris, Calmann-Lévy.

ouologuem, Y., 1968 Le devoir de violence, Paris, Éditions du Seuil.

SARTRE, J.-P., 1948 « Orphée noir », Préface, in L. P. SENGHOR, Anthologie de la nouvelle poésie nègre et malgache de langue française, Paris, Presses universitaires de France.

SENGHOR, L. S., 1948 Anthologie de la nouvelle poésie nègre et malgache de langue française, Paris, Presses universitaires de France.

-, 1959 « Éléments constructifs d'une civilisation d'inspiration négro-africaine », Présence Africaine, 24-25 : 71-72.

—, 1996 « Les leçons de Leo Frobenius », Présence Africaine, 117 : 178-179.

SIBEUD, E., 2002 Une Science impériale pour l'Afrique ? La construction des savoirs africanistes en France 1878-1930, Paris, Éditions de l'École des hautes études en sciences sociales.

\section{NOTES}

1. À savoir, "un système de dispositions durables et transposables, structures structurées prédisposées à fonctionner comme des structures structurantes, c'est-àdire en tant que principes générateurs et organisateurs de pratiques et de représentations qui peuvent être objectivement adaptées à leurs buts ».

2. Voir également Jean BAZIN (1985 : 92-94). Selon lui, «À la différence du peuple ou de la nation, produits d'une histoire, l'ethnie est en effet le résultat d'une opération préalable de classement [...]. Qu'on croit ou non à sa réalité substantielle, l'ethnie est ce sujet fictif que l'ethnologie contribue à faire être, le perpétuant comme entité de référence dans un espace savant, grâce à ses procédures inductives et attributives » (ibid.). 
3. Sur l'œuvre du baron Roger, voir ma présentation (AGGARWAL 2007, 2008).

4. Voir sa préface dans AMSELLE et SIBEUD (1998: 5).

5. Instructif également de noter que pour les fondateurs de l'Institut, l'africanisme est un prétexte devant contribuer à légitimer un impérialisme de gauche susceptible de contrecarrer l'influence croissante du communisme. L'Institut avait donc le mandat de former «des conservateurs qualifiés qui seront aussi les experts légitimes d'une politique indigène conforme aux principes républicains ». Voir Emmanuelle SIBEUD (2002 : 266).

6. La biographie de Louise DELAFOSSE (1976 : 375-388) fournit d'importantes informations quant à la position de son père au sein des réseaux africanistes en Europe.

7. Il va de soi que les propos du linguiste démystifiait l'idée que tenait à promouvoir Senghor de la nature et de la fonction de l'oralité en Afrique noire puisque, à son sens, «L'oralité n'est pas l'absence ou la privation de l'écriture. Elle se définit positivement comme une technique et une psychologie de la communication » (HouIs 1971 : 9).

8. Sur la relation entre la démarche de Griaule et celle de Hampâté Bâ, se référer à $K$. AGGARWAL (1999 : 78-106).

9. Voir à ce sujet, Pascale CASANOVA (1999: 110-118). «La révolution herderienne » renvoie ici au nouveau rapport institué entre nation et langue, à l'instar des théories du philosophe allemand Herder. Se fondant sur la notion de "génie » et d' "âme ", il invente un mode d'accumulation symbolique qui permet aux pays politiquement dépendants d'accéder à l'égalité et donc à la dignité culturelle.

10. C'est là sans doute une approche originale qui paraît se situer en continuité avec le questionnement amorcé par Paul GILROY (1993) sur le statut de l'Atlantique noire comme lieu de l'insertion des peuples noirs dans la modernité occidentale.

11. Le critique français développe ici une analyse éclairante de la problématique de la littérature migrante en se basant sur l'analyse de la notion de "l'énigme de l'arrivée » développée par V.S. Naipaul dans son roman du même nom inspiré du tableau éponyme de Giorgio De Chirico, confrontant les antinomies de la linéarité du voyage à la circularité de l'éternel retour.

\section{RÉSUMÉS}

Résumé

Paradoxalement, l'africanisme occidental, considéré en règle générale comme «la fille de l'impérialisme colonial», peut se concevoir également comme "le père fondateur de la littérature africaine moderne" dans la mesure où ces deux domaines de réflexion et de recherche sur l'Afrique s'enchaînent et s'entrecoupent systématiquement, et cela à plusieurs niveaux.

Cet article a pour objet de souligner les filiations. Il s'organise en trois temps et interroge successivement le discours élaboré sur les sociétés et les cultures africaines depuis l'émergence de l'africanisme occidental jusqu'à la naissance, dans l'après-guerre, d'une littérature de langue 
française proprement africaine qui tente de créer une littérature-monde et d'inventer ainsi une nouvelle écriture africaine susceptible de transcender les contraintes de l'immobilité associées aux notions de territorialité et d'appartenance identitaire. Au-delà de l'analyse des perspectives africanistes développées dans le cadre de la recherche coloniale, on sera attentif à la façon dont certains écrivains et penseurs africains, de Senghor à Miano, de Hampâté Bâ et Mudimbe à Mbembe et Mabanckou, perçoivent leur relation à l'Afrique et à la notion de l'africanité. Au terme de cette analyse, on constatera effectivement que la prise en compte de l'africanisme occidental peut ouvrir de nouvelles perspectives pour comprendre la littérature africaine et l'enrichir.

Abstract

Paradoxically, Western Africanism, though usually defined as the "daughter of colonial imperialism" may also be construed as "the founding father of modern African writing in French" since these two areas of thought and knowledge intersect and overlap systematically at several levels.

This essay conceived with the primary objective of delineating interconnections that bind these two discursive constructions, is organized in three broad sections and successively interrogates discourses dealing with African societies and cultures from the time of its inception with the advent of Western Africanism until the rise of African writing in French during the post-war years, and the current endeavors at creating a World Literature and inventing a new literary idiom in a bid to transcend constraints of immobility associated with notions of territoriality and belonging. Other than Africanist perspectives developed in the context of colonial research, this essay will closely look at the ways in which African writers and thinkers from Senghor to Miano, from Hampâté Bâ and Mudimbe to Mbembe and Mabanckou perceive their relationship with Africa and ideas of Africanity. At the close of this reading, what may perhaps appear with some clarity is that an understanding of Western Africanism is crucial for an understanding of African literature, for not only does it hold the possibility of providing new critical perspectives, it also inhibits recourse to binaries that often tend to dominate interpretations of African literary works.

\section{INDEX}

Mots-clés : africanisme, africanité, littérature africaine, littérature-monde, oralité, postcolonie, savoir occidental, subjectivité

Keywords : Africanism, Africanity, African Literature, World Literature, Orality, Post-Colonial, Western Knowledge, Subjectivity

\section{AUTEUR}

\section{KUSUM AGGARWAL}

Department of Germanic and Romance Studies, University of Delhi, Delhi, Inde. 